\title{
Memory processing of spatial order as transmitted by auditory information in the absence of visual cues
}

\author{
MARCO W. BATTACCHI, ANGELO FRANZA, and ROBERTO PANI \\ Institute of Psychology, Institute of Educational Sciences, University of Bologna, Bologna, Italy
}

\begin{abstract}
Two experiments were conducted concerning spatial order recall when spatial information is transmitted by auditory stimuli. Temporal order either was congruent with spatial order or was independent of spatial order. In Experiment 1, the comparisons were among normally or partially sighted subjects allowed to look, normally sighted subjects who were blindfolded, and blind children. The main findings were a superiority of the sighted subjects allowed to look (that is, to support auditory information with visual cues) and a smaller advantage for the sightedbut-blindfolded subjects, relative to the blind group. In Experiment 2, normally sighted adults (either seeing or blindfolded) and blind adults were tested. Surprisingly, the blind were not worse than the sighted in this study. Subsequent interviews and detailed analysis of errors suggested that the blind coded spatial information kinesthetically. These indirect analyses also suggested that whereas spatial order was coded temporally in the sighted, it was controlled by both temporal and spatial factors in the blind and blindfolded subjects.
\end{abstract}

The blind are obviously deprived of the dominant modality for spatial information processing. It has been convincingly demonstrated that space is primarily represented in visual terms and that the visual system is especially suitable for dealing with spatial information (O'Connor \& Hermelin, 1978). The blind, of course, employ other spatial senses (touch, hearing, proprioception, and kinesthesis) to take the place of vision.

Research has focused on vicarious processing of space information in the blind by means of tactile and kinesthetic cues, whereas the use of auditory cues has very seldom been studied. In the experiments reported here, we tried to study immediate memory ${ }^{1}$ for spatial order when input is auditory and made up of verbal stimuli, that is, in conditions in which verbal or auditory-articulatory coding is facilitated (O'Connor \& Hermelin, 1978).

\section{EXPERIMENT 1}

We aimed at studying the influence of temporal distribution on spatial order recall of verbal stimuli in sighted seeing, sighted blindfolded, partially sighted, and blind subjects. Previous research in this field has shown that when the to-be-remembered

\footnotetext{
This work was supported by C. N. R. Grant CT 79.00489.08. The authors are indebted to Maria Grazia Galetto and Edda Paltrinieri for their help in conducting these experiments. We also appreciate the valuable suggestions of Alice F. Healy and Neal O'Connor. The senior author is indebted to the NATO Programme on Human Factors for a Study Visit Award to Yale University.
}

material is verbal (regardless of whether it is heard or seen), incongruent temporal and spatial distributions yield poorer performances than do congruent ones (Anderson, 1976; Mandler \& Anderson, 1971). Two conflicting explanations have been put forth: (1) Temporal and spatial structures are independent and additive retrieval cues, and when they are congruent, they reinforce each other (Anderson, 1976; Mandler \& Anderson, 1971); (2) spatial order recall is a temporally mediated process, either in the sense that spatial order information is the content of the store and some sort of temporal ordering is the address to the store (Murdock, 1969), or in the sense that spatial order information is translated into a temporal sequence of spatial positions ("temporalspatial patterns,"' according to Healy, 1975a, 1977, 1978). In the case of incongruent distributions, reconstruction from temporal ordering, or coding about temporal-spatial patterns, may be too difficult because of the high information load (temporal and spatial distributions of items are not redundant).

At any rate, spatial order recall of sequentially presented verbal material is better when presentation is auditory than when it is visual (Murdock, 1969), and temporal order recall is better than spatial order recall only when the number of items per list is too great and/or the temporal order is not kept constant across trials (Healy, 1975a, 1975b, 1977, 1978).

The explanation for the first finding could be related to an inherent superiority of auditory shortterm memory (Murdock, 1969). The explanation for the second finding would then be that pure spatial order recall is studied only under constant temporal order of items (Healy, 1978) and only when the 
number of alternative temporal-spatial patterns is not too great.

All the evidence so far reviewed comes from research carried out on normally sighted adults allowed the use of their eyes, but what about blind and blindfolded subjects? Some predictions can be inferred by indirect evidence. In particular, the blind should be inferior to the sighted blindfolded, and both of these groups should be inferior to sighted seeing subjects. Indeed, pointing at an invisible sound source is much more accurate with the eyes open than with the eyes closed (Warren, 1970), and eye movements combine with visual information to fix auditory position (Platt \& Warren, 1972). Further, the blind make more errors than the sighted blindfolded when trying to establish the absolute position of a sound source (Gomulicki, 1961, cited in O'Connor \& Hermelin, 1978). According to Warren (1970), visual mapping of auditory stimuli provides an optimal strategy for locating sounds in space; having one's eyes open seems to maximize such visual mapping, but the sighted, even with their eyes closed, have an advantage over the blind (O'Connor \& Hermelin, 1978). The evidence refers to locating sound sources, but it is likely that these difficulties hold even more for retention of spatial location, and especially when retention of order (i.e., of the relative locations of a series of stimuli) is tested. Furthermore, blindfolded children have shown an advantage over blind children of the same age in a variety of tactile-kinesthetic discrimination and memory tasks (see review by O'Connor \& Hermelin, 1978), and that advantage has been explained as being due to the availability of a visual map in the blindfolded subjects.

A third prediction is that the partially sighted, even though they have no direct evidence available to them, will give better overall performance than the blind, due to their less severe visual impairment and their ability to draw a visual representation of space, however rough it may be. With regard to the two presentation conditions, there is every reason to expect an advantage of the congruent condition over the incongruent one: If information is verbally rehearsed, it is coded in auditory-articulatory (i.e., temporal) terms, and this coding should interfere with spatial coding when temporal and spatial distributions are incongruent. If no auditory-articulatory coding is employed in spatial order recall, but rather a coding of temporal-spatial patterns is used, as Healy $(1975 a, 1977,1978)$ maintains, the information load is greater in the incongruent condition than in the congruent condition.

Finally, we expect the advantage of congruency to be even greater for visually impaired and visually restricted subjects, if space is primarily processed in visual terms, either because it is too difficult or impossible to contrast temporal coding by visual cues or because the spatial component of temporal-spatial patterns is too weak or null.

\section{Method}

Material and Design. The paradigm of Murdock's (1969) Experiment 3 was used. Six-item lists were made of Italian twosyllable proper male names (Gigi, Luca, Mario, Nello, Pino, and Vito) by arranging the six names in different random orders. Each name issued from one of six loudspeakers placed in a semicircle around the subject. At every trial, after the presentation of the list, the experimenter, who was located behind one of the loudspeakers, asked which name had issued from that loudspeaker (probe technique), and the same procedure was repeated for a second location. Thus, two different locations were probed on each trial, but all the locations were evenly tested, with the restriction that each location be tested first and second equally often.

Two conditions were considered: In the congruent temporalspatial distribution, the order of the names in time corresponded to their order in space (first in time = first from the subject's right, and so on). In the incongruent temporal-spatial distribution, the relationship between order in space and order in time was random, in such a way that neither of the two orders was constant across trials. In the congruent condition, the right-to-left order was chosen because in a preceding experiment (Battacchi, Franza, \& Garbesi, 1977) on temporal order recall, we found a slight advantage for this order, perhaps related to hemispheric dominance. There were 24 lists per condition and 48 trials per subject. For each group of subjects, if the first subject had started to recall in the congruent condition, the second subject started in the incongruent condition, and so on, and if the first block of 24 lists had been assigned to the congruent condition for the first subject, it was assigned to the incongruent condition for the second subject, and so on.

Procedure. The experiment was performed in a soundproofed room measuring $220 \times 360 \times 325 \mathrm{~cm}$ : On one wall was located a tape recorder (Model $3002 \mathrm{Hi}$-Fidelity Telefunken) and a special selector, purposely devised for this experiment. This selector, equipped with directional indicators, allowed for the channeling (through its 12 output channels) of the auditory stimuli that it received in accordance with the experimenter's schedule. In this particular case, lists of six two-syllable proper Italian names had been taped beforehand, to be transmitted by the experimenter through the use of the selector. These names were preceded by an extremely sensitive acoustic signal that the selector was able to pick up. When "play" was pressed on the tape recorder, the taped stimulus triggered the release mechanism on the selector. This, in turn, channeled the sound of the six recorded names into six loudspeakers to which the tape recorder was connected. Therefore, the release mechanism was automatic, and as such, it was programmed to click every $1 \mathrm{sec}$; as a result, the six names were transmitted through the speakers within $1 \mathrm{sec}$ of each other. The wooden speakers were specially made and were placed in movable vertical stands. The subject was asked to sit in a comfortable chair in the center of the room and to concentrate on the experiment to the best of his ability. The subject was told that it was simply an experiment that hoped to "shed light" on the mechanisms involved in a person's spatial-temporal orientation. The subject was also told that besides the possibility of making an outright mistake in his answers, it was also possible that he would be unable to remember, and that this, again, was no cause for worry.

The subject was seated in a comfortable chair in the center of the room at a distance of approximately $180 \mathrm{~cm}$ from the six speakers, which were arranged in a semicircle around him, $75 \mathrm{~cm}$ apart from each other. The semicircle covered approximately $480 \mathrm{~cm}$. The speakers were adjusted to a comfortable level for each observer. When the experiment involved a blind subject, the spatial disposition of the speakers was described to him, and, if 
necessary, he was encouraged to touch the speakers or to move about the room so as to be able to mentally reconstruct the entirety of the environment. The same was done for partially blind subjects.

The presentation of the successive lists started as soon as the subjects had finished recalling the preceding one, and it was always signaled by the word "ready," spoken by the experimenter.

Subjects. There were four groups: 10 normally sighted seeing children, 10 partialiy sighted children (visual acuity $=1 / 10$ or less), 10 normally sighted blindfolded children, and 10 congenitally blind or nonvisualizing children (age range $=8-10$ years) Blind and partially sighted children were chosen from the population of a special institution; normally sighted children came from the population of a primary school. The groups were matched for age, school grade, and sex.

Scores. Number of correct responses was the main measure, scored under both a strict criterion (number of names recalled in the right location) and a weak criterion (number of names rightly placed and/or misplaced by only a one-place error). The weak criterion was added in order to rule out the perceptual effect of poor localization without vision. Of course, any "blank" response was counted as an error under both criteria.

\section{Results}

Results are presented in Table 1 as percentages of correct responses. An analysis of variance for a mixed design with one within-subjects factor and one between-subjects factor was performed on the raw data.

The overall difference between conditions was statistically significant and in the expected direction for both the strong and the weak criteria $[F(1,36)=$ $8.65, p<.01$, and $F(1,36)=47.75, p<.01$, respectively]. Performance was superior in the congruent condition.

The overall differences between groups were statistically significant for both scoring criteria $[F(3,36)=14.75, \quad p<.01$, and $F(3,36)=10.12$, $\mathrm{p}<.01$, respectively]. All pairs of group means were compared by using the Newman-Keuls test. As expected, normally sighted seeing subjects were significantly superior, for both criteria, to the blind as well as to the sighted blindfolded and the partially sighted $(p<.01)$. There was no significant difference between the sighted blindfolded and the blind with the strong criterion. Indeed, both groups performed at about chance level (since two positions were tested at each trial, the chances for at least one correct response per trial were equal to $1 / 3$, or $33.33 \%$ ). On the other hand, the difference was significant and in the expected direction for the weak criterion $(p<.05)$. The partially sighted were superior to the blind for both criteria $(p<.05)$.

The Groups by Conditions interaction was significant only for the weak criterion $[F(3,36)=3.37$, $\mathrm{p}<.05]$. In order to explain the interaction, a $t$ test for the difference between conditions was carried out for each group. Only the differences for the sighted seeing, regardless of whether the subjects were normally or partially sighted, were significant (for the normally sighted, $t=4.823, p<.01$; for the partially sighted, $t=2.312, p<.025$ ). Contrary to the hypotheses, the visual-input restricted subjects showed no significant difference between conditions, whereas the sighted subjects did.

\section{Discussion}

The superiority of spatial recall when temporal and spatial distributions are congruent has been confirmed. The predicted superiority of the normally sighted over the partially sighted and of both of them over the blind has been supported. The internal visual map hypothesis, as opposed to the visual-input restriction hypothesis, which would have been supported by a superiority of the blindfolded over the blind, was not supported when a strong criterion of scoring was adopted, but it was supported when performance was scored on the basis of a weak criterion.

These findings are not really contradictory, if one considers the special requirements of the experimental task. The probe technique not only called for the retention of spatial order (i.e., of the relative location of each item within the set), but also for the retention of the position of each item relative to a frame of reference external to the set of items ("absolute" location). In other words, subjects had to retain not only that a given item was the first one from the extreme right, but also that it was "right there," in that region of space. This kind of task is rather exacting, especially if subjects cannot rely upon any visual frame of reference or on any actual visual cue. The availability of an internal visual representation of space succeeds in showing its positive influence if only a broad localization, as that afforded by a weak scoring criterion, is required. In conclusion, space processing is superior when a

Table 1

Percentages of Correct Responses (Experiment 1)

\begin{tabular}{lccccccc}
\hline & \multicolumn{4}{c}{ Temporal-Spatial Order } \\
\cline { 2 - 6 } & Congruent & Incongruent & Total & Congruent & Incongruent & Total \\
\cline { 2 - 6 } & 35.00 & 30.42 & 32.71 & 58.33 & 50.42 & 54.37 \\
Blind & 34.58 & 31.04 & 32.81 & 66.87 & 61.04 & 63.96 \\
Blindfolded & 43.75 & 38.12 & 40.94 & 66.46 & 58.12 & 62.29 \\
Partially Sighted & 59.37 & 45.21 & 52.29 & 81.25 & 63.54 & 72.39 \\
Seeing & & & & &
\end{tabular}


visual map is available, but it is even better when visual input is actually accessible. The same conclusion was drawn from the studies on the ability to locate sounds in the dark or those issued from a hidden source (Warren, 1970).

The findings regarding the interactions can be explained in the same way. Contrary to our expectations, the more visually impaired the subjects were, the less was the difference between conditions. Apparently, the task of retaining the absolute position of each item was so demanding that it did not matter whether temporal and spatial distributions were congruent or not. The explanation offered was in terms of memory processes (retention of spatial positions) and not in perceptual terms (localization of items in space), because the likelihood of localization errors was greatly reduced by the weak scoring criterion. In point of fact, only the findings that were significant also, or only, under the weak criterion were taken into consideration.

Further results. The data can be analyzed from a different point of view and for a different purpose. Murdock (1969) supported his contention that retention of spatial order was mediated by temporal order by comparing error distributions in time and space. He found that temporal gradients showed the adjacency effects that are characteristic of serial tasks (i.e., the closer in time the target response and the response substituted for it were, the more frequent was the substitution), whereas spatial gradients were essentially flat (i.e., a misplacement of, say, two spatial positions was not more frequent than a misplacement of three or four positions).

Anderson (1976) also found spatial gradients to be flatter than temporal gradients. Substitution errors were controlled only by temporal adjacency, and spatial order recall was controlled by temporal distribution. A similar analysis was made on our data for the incongruent condition. Results are shown in Table 2 as the differences between observed and expected frequencies, expressed as percentages of total substitution errors. A " +" score means that the observed frequency was greater than the proportion of the overall errors expected for that n-position misplacement if errors had been determined by chance; a " -" score is for the opposite relationship. The expected frequencies were calculated by con- sidering that in a six-position series, there are 32 chances of item displacement, of which 10 are for a one-position misplacement, 8 for a two-position misplacement, 6 for a three-position misplacement, 4 for a four-position misplacement, and 2 for a fiveposition misplacement.

Spatial gradients were flatter (i.e., the deviations from the expected values were lower) for the spatial gradients than for the temporal gradients in the sighted seeing, both normally and partially sighted, in accordance with Murdock's (1969) findings. However, surprisingly enough, the blind and the blindfolded showed a nearly flat distribution for the temporal (and not for the spatial) gradient. If a flat distribution for a given dimension means that substitution errors are not controlled by it, a nonflat distribution for that dimension should mean that substitution errors do depend on it. Spatial order recall therefore seems to be mainly temporally mediated in the sighted seeing and spatially controlled in the visual-input restricted. Any interpretation of this interesting finding will be postponed for the general discussion, which follows the results of the next experiment.

\section{EXPERIMENT 2}

Experiment 1 was replicated with older subjects, who were highly skilled in verbal behavior and strategies of verbal rehearsal. The need to check the generalization limits of the results obtained in Experiment 1 was motivated by the dramatic difference in temporal order recall between children and older subjects, as was found in previous experiments on temporal order recall (Battacchi et al., 1977; Battacchi, Franza, \& Pani, in press). On the other hand, there is no reason to change the set of the previously formulated hypotheses in a substantial way. The overall difference between conditions should remain. As for the overall differences between groups, the high verbal skills of all the subjects allow for the prediction of a general improvement in recall performance and possibly a reduction of betweengroup differences, should the general improvement be so great as to cause ceiling effects. As for the interactions, the results of Experiment 1 lead to the tentative prediction that the differences between con-

Table 2

Gradients of Substitution Errors: Differences Between Observed and Expected Frequencies (Experiment 1)

\begin{tabular}{|c|c|c|c|c|c|c|c|c|c|c|}
\hline & \multicolumn{10}{|c|}{ Misplacement } \\
\hline & \multicolumn{2}{|c|}{ One Position } & \multicolumn{2}{|c|}{ Two Positions } & \multicolumn{2}{|c|}{ Three Positions } & \multicolumn{2}{|c|}{ Four Positions } & \multicolumn{2}{|c|}{ Five Positions } \\
\hline & Spatial & Temporal & Spatial & Temporal & Spatial & Temporal & Spatial & Temporal & Spatial & Temporal \\
\hline Blind & +7.0 & +1.5 & +3.0 & -2.0 & -6.5 & +.5 & -3.0 & +.5 & -.5 & -.5 \\
\hline Blindfolded & +13.5 & +2.5 & -5.0 & +1.5 & -4.5 & -3.0 & -1.0 & +2.0 & -3.0 & -3.0 \\
\hline Partially Sighted & +8.0 & +14.0 & -2.5 & -2.0 & -5.0 & -2.0 & +1.0 & -6.0 & -1.5 & -4.0 \\
\hline Seeing & +5.0 & +12.5 & -3.0 & +3.5 & +1.0 & -5.0 & -1.5 & -8.0 & -1.5 & -3.0 \\
\hline
\end{tabular}


ditions should be smaller for the visually impaired groups.

\begin{abstract}
Method
Eight nonvisualizing blind, eight normally sighted seeing, and eight normally sighted blindfolded individuals were the subjects. A sample of partially sighted subjects was dropped from the experiment because the preceding experiment showed that the partially sighted behave just as sighted seeing subjects with low discriminatory power. All the subjects were university students (age range $=21-25$ years). The normally sighted were randomly assigned either to the seeing group or to the blindfolded group. All the subjects had volunteered for the experiment.
\end{abstract}

\section{Results}

The results are presented in Table 3 as percentages of correct responses. The usual advantage for the congruent condition over the incongruent condition was confirmed for data scored under both criteria $[F(1,21)=121.26$ and $F(1,21)=73.84$, respectively; in both cases, $\mathrm{p}<.01]$. The predicted general improvement in recall performance of adults compared with children was also confirmed (see Tables 1 and 3).

The differences between groups were significant according to both scoring criteria $[\mathrm{F}(2,21)=8.88$ and $F(2,21)=89.75$, respectively; in both cases, $p<.01$, but the striking result is that there was no significant overall difference between the blind and the normally sighted seeing. Only the sighted blindfolded were inferior to both of the other groups (compared with the sighted seeing, $\mathrm{p}<.01$ under both criteria; compared with the blind, $\mathrm{p}<.05$ under the strong criterion and $p<.01$ under the weak criterion; Newman-Keuls test). The Conditions by Groups interaction was not significant. Apparently, the group and the condition factors acted independently.

Introspective data. The interviews held after the completion of the experimental task gave quite interesting results: All subjects were aware of their efforts to disregard temporal order, even in the congruent condition. The spatial strategies adopted were of two sorts, sometimes combined. The first was dividing the space into broad regions (e.g., right and left) and trying to fill the regions in with the specific item positions; the second, used for the incongruent condition, was mentally constructing patterns that would link several item positions. Both strategies were common to the sighted as well as to the blind, but the modality in which the patterns were represented was different. The sighted said they tried to create visual geometric forms, whereas the blind tried to create motor patterns by imagining, for example, the linking of several positions by a thread.

These introspections lead to some interesting conjectures. In the first place, they confirm Healy's (1975a) contention of an independent, non-auditoryarticulatory coding of information in spatial order recall. Second, motor pattern representation is actually a temporal-spatial coding. For example, connecting the first (temporally) three items by an imagined thread going from the second spatial position of the first item to the fourth spatial position of the second and then turning back to the first spatial position of the third (spatial positions ordered leftward) is a sort of temporal-spatial coding, as was put forth by Healy. We are suggesting that the blind coded spatial order in motor terms, as actual kinesthetic patterns or patterned motor programs. The relative failure in such a coding was probably due to the great load imposed on working memory by our relatively long sets of items (see Healy, 1977).

Gradients of substitution errors. The spatial gradients of substitution errors were nearly flat, at variance with the temporal gradient, for the sighted seeing, whereas the peak of substitution errors at the one-position misplacement was higher on the spatial gradient than on the temporal one for both the blind and the sighted blindfolded (i.e., for the visual-input restricted) (see Table 4). Once again, temporal adjacency was more important than spatial adjacency for the sighted seeing, whereas the converse relationship held for the visual-input restricted.

Serial position functions. The distribution of substitution errors on the spatial positions is shown in Figures 1 and 2. The data are expressed as percentages of the overall substitution errors for each group and for each condition, so that the curves are directly comparable. A three-factor analysis of variance for a mixed design with two within-subjects factors (congruent vs. incongruent condition and serial position) and one between-subjects factor (experimental groups) was performed (Winer, 1962). In addition to the main factors (groups, conditions, and serial positions), only the Condition by Serial Position interaction was significant $[F(5,105)=13.07$,

Table 3

Percentages of Correct Responses (Experiment 2)

\begin{tabular}{lcccccc}
\hline & & \multicolumn{3}{c}{ Temporal-Spatial Order } & & \multicolumn{2}{c}{ Weak Criterion } \\
\cline { 2 - 6 } & Congruent & Incongruent & Total & Congruent & Incongruent & Total \\
\hline Blind & 64.0 & 46.0 & 55.1 & 83.6 & 71.2 & 77.7 \\
Blindfolded & 53.6 & 35.7 & 44.7 & 78.4 & 64.6 & 71.5 \\
Seeing & 73.7 & 47.9 & 60.1 & 89.3 & 67.7 & 78.5 \\
\hline
\end{tabular}


Table 4

Gradients of Substitution Errors: Differences Between Observed and Expected Frequencies (Experiment 2)

\begin{tabular}{|c|c|c|c|c|c|c|c|c|c|c|}
\hline & \multicolumn{10}{|c|}{ Misplacement } \\
\hline & \multicolumn{2}{|c|}{ One Position } & \multicolumn{2}{|c|}{ Two Positions } & \multicolumn{2}{|c|}{ Three Positions } & \multicolumn{2}{|c|}{ Four Positions } & \multicolumn{2}{|c|}{ Five Positions } \\
\hline & Spatial & Temporal & Spatial & Temporal & Spatial & Temporal & Spatial & Temporal & Spatial & Temporal \\
\hline Blind & +19.0 & +17.0 & -7.0 & -3.0 & -5.0 & -5.0 & -6.0 & -5.0 & -1.0 & -4.0 \\
\hline Blindfolded & +17.0 & +12.0 & -2.5 & +2.0 & -7.5 & -7.0 & -6.0 & -3.5 & -1.0 & -3.5 \\
\hline Seeing & +6.5 & +20.0 & +.5 & -4.0 & -1.0 & -8.0 & -5.0 & -5.5 & -1.0 & -2.5 \\
\hline
\end{tabular}

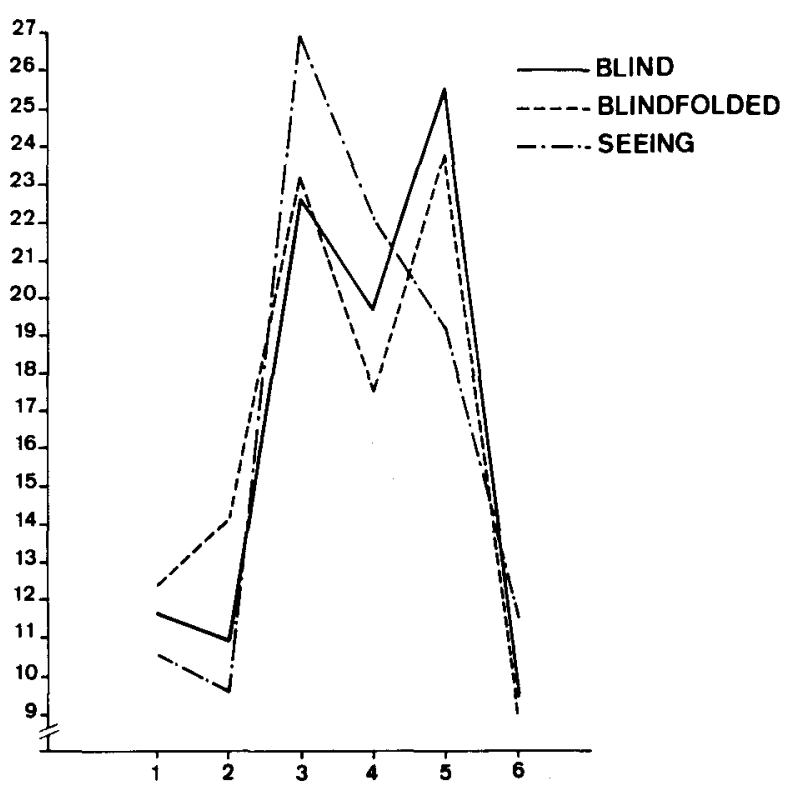

Figure 1. Spatial serial curves (normalized) for the congruent condition (percentages of the overall errors).

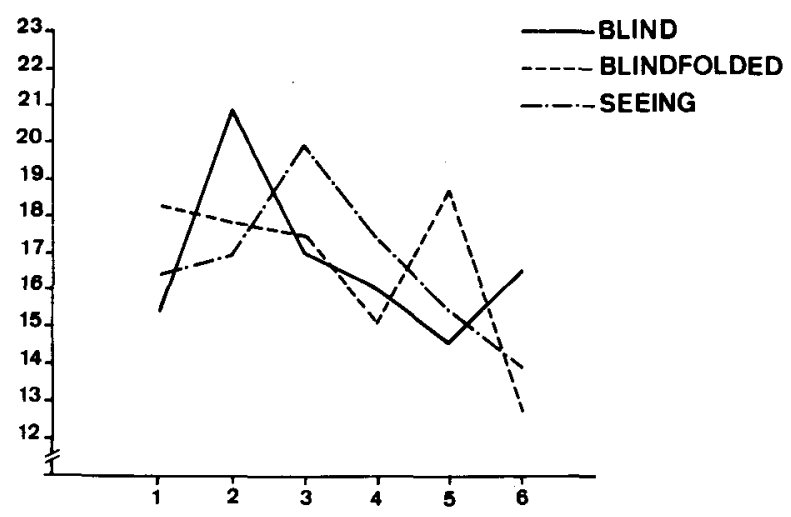

Figure 2. Spatial serial curves (normalized) for the incongruent condition (percentages of the overall errors).

$p<.01]$. Inspection of the data shows that the classic bow-shaped curve was obtained only for the congruent condition; this result was true across the groups.

These results confirm Healy's (1975a, 1977) findings and agree with the predictions derived from
Estes' (1972) synchrony model, according to which the bowed functions would not be observed with spatial rather than temporal positions in a situation in which the temporal and spatial positions are varied independently, whereas the bowed curves would be expected for temporal positions even when subjects are to recall the spatial order of items. Apparently, the bowed curves obtained for the congruent condition depend on temporal order processing.

\section{GENERAL DISCUSSION AND CONCLUSION}

The most important result was the improvement in spatial recall performance of young blind adults compared with that of blind children, which allowed the adults to equal normally sighted seeing subjects of the same age. The most obvious explanation would refer to the improvement in verbal rehearsal with age (Anderson, 1976), but the blindfolded adults did not show any improvement of this kind, even though they too were superior to the blindfolded children in verbal rehearsal. Introspective data suggest that the improvement was due to the development of effective strategies of kinesthetic coding that were not likely to be available to blind children. Indeed, blind children are able to draw effectively on kinesthetically derived images only for very short sequences (O'Connor \& Hermelin, 1978). This post hoc explanation is worth taking as a hypothesis to test with specifically devised experiments. According to Attneave and Benson (1969), spatial information from modalities other than vision is transferred, if possible, to an internal and more "appropriate" visual representation of space. However, here, we are claiming that spatial information from the auditory modality is transferred, by the blind, to an internal kinesthetic representation of space and that this representation seems to be as appropriate as the visual one. Furthermore, the efficiency of this process is quite remarkable if one considers that a kinesthetic coding allows only for the construction of a projective and not a euclidean space (O'Connor \& Hermelin, 1978). Along the same lines, the shift from the superiority of blindfolded 
children over blind children to the superiority of blind adults over blindfolded adults can be explained by assuming that the advantage given to the blindfolded by visual mapping is overcome by the poor development of kinesthetic strategies in subjects who are not accustomed to being visual-input restricted.

The results of Experiment 2 are quite consistent with Murdock's (1969) findings as far as the sighted seeing are concerned: From the analysis of substitution errors, it appears that spatial order recall was controlled by temporal factors (temporal adjacency of items and distance from the temporal end positions). Murdock's theory, according to which spatial information is extracted through a temporally mediated reconstruction, was supported. On the other hand, for the visual-input restricted, spatial order recall was controlled by temporal factors (distance from the temporal end positions), as well as by spatial factors (spatial adjacency).

These results can be explained by assuming that the sighted seeing are generally able to represent the exact "absolute" location of each item, and their substitution errors depend on the retrieval processes through temporal cues or on the reverberatory mechanisms preserving the successively displayed items (Estes, 1972). On the other hand, for the visualinput restricted, some minor item displacements may depend also on a coarser representation of items' "absolute" locations. The greater proportion of oneposition misplacements for the blind $(53 \%$ of total errors) and for the blindfolded (51\%) than for the sighted seeing $(40 \%)$ might be the result of such a less powerful spatial processing, and the opposition of kinesthetic and visual coding might be responsible for it. In this case, the recall processes for spatial order of the visual-input restricted would not be basically different from those of the sighted seeing: Recall would be a temporally mediated reconstruction of the order of items whose locations were loosely represented. Some indirect evidence is given by the peak at the one-position misplacement in the temporal gradient that was found even for the visualinput restricted.

This explanation rules out the possibility that the improvement of blind adults over blind children may be entirely due to a better representation of item location. The spatial gradients of both groups are quite similar, and presumably, both groups had the same sort of difficulty in retaining item location.
If so, then the improvement in recall performance of blind adults should depend on the development of more effective strategies for retention of spatial order.

\section{REFERENCES}

ANDERSON, R. E. Short-term retention of the where and when of pictures and words. Journal of Experimental Psychology: General, 1976, 105, 378-402.

Attneave, F., \& Benson, L. Spatial coding and tactual stimulation. Journal of Experimental Psychology, 1969, 81, 216-222.

Battacch1, M. W., Franza, A., \& Garbesi, P. Spatial reference and serial recall in blind, partially sighted and normal children. Italian Journal of Psychology, 1977, 4, 285-297.

Battacchi, M. W., Franza, A., \& Pani, R. Spazio e tempo nella memoria immediata. Psychologica, in press.

Estes, W. K. An associative basis for coding and organization in memory. In A. W. Melton \& E. Martin (Eds.), Coding processes in human memory. Washington, D.C: Winston, 1972.

Healy, A. F. Coding of temporal-spatial patterns in short-term memory. Journal of Verbal Learning and Verbal Behavior, 1975, 14, 481-495. (a)

HeAly, A. F. Short-term retention of temporal and spatial order. Bulletin of the Psychonomic Society, 1975, 5, 57-58. (b)

Healy, A. F. Pattern coding of spatial order information in short-term memory. Journal of Verbal Learning and Verbal Behavior, 1977, 16, 419-437.

HEALY, A. F. A Markov model for the short-term retention of spatial location information. Journal of Verbal Learning and Verbal Behavior, 1978, 17, 295-308.

Mandler, G., \& Anderson, R. E. Temporal and spatial cues in seriation. Journal of Experimental Psychology, 1971, 90, 128-135.

Murdock, B. B. Where and when: Modality effects as a function of temporal and spatial distribution of information. Journal of Verbal Learning and Verbal Behavior, 1969, 8, 378-383.

O'Connor, N., \& Hermelin, B. Seeing and hearing and space and time. London: Academic Press, 1978.

Platt, B. B., \& Warren, D. H. Auditory localization: The importance of eye movements and a textured visual environment. Perception \& Psychophysics, 1972, 12, 245-248.

WARREN, D. H. Intermodality interactions in spatial localization. Cognitive Psychology, 1970, 1, 114-133.

WINER, B. J. Statistical principles in experimental design. New York: McGraw-Hill, 1962.

\section{NOTE}

1. We shall refer to immediate memory as it pertains to the sort of experimental technique we used (i.e., immediate recall after presentation of each list of items)

(Received for publication July 14, 1980; revision accepted November 17,1980 .) 